\title{
569 A NOVEL MICROBIOME-DERIVED PEPTIDE REVERSES RESISTANCE TO ANTI-PD-1 THERAPY
}

Dhwani Haria*, Jayamary Divya Ravichandar, Jill Desnoyer, Sabina Lau, Jina Lee, Erica Rutherford, Preeti Lal, Karim Dabbagh, Helena Kiefel. Second Genome, Inc, Brisbane, CA, USA

Background Despite the unprecedented clinical success of immune checkpoint therapy (ICI) in many cancers there is still a large unmet need since many patients have an inadequate response. Fecal Matter Transplant from anti-PD-1 responding subjects into non-responders improves response in many subjects, providing a strong rationale that the gut microbiome has a critical role in modulating effects of ICI therapies. However, the causative mechanisms and host targets that mediate these beneficial therapeutic effects are still poorly understood. The objective of the present study was to demonstrate that peptides derived from the microbiome of ICI responders, have the potential to bring a similar therapeutic benefit to ICI non-responders via direct immunomodulatory effects through a host target.

Methods Using Second Genome's proprietary algorithms, we derived a microbial signature that was enriched in responding subjects across multiple cohorts. The peptides identified from these strains were screened in a classical drug discovery pipeline to discover peptides that modulate the human immune system by secreting proinflammatory cytokines and chemokines such as CXCL10 and TNF- $\square$ by human dendritic cells (DCs), and mediate anti-tumor immunity and improve response to anti-PD-1 therapy in mouse tumor models.

Results We found several peptides which bind primary human cells, exert immune function, and exert anti-tumor effects. Particularly, peptide SG-3-00802 showed improved anti-tumor responses in combination with anti-PD-1 in the anti-PD-1 insensitive RENCA model. Treatment with SG-3-00802, alone or in combination with anti-PD-1 significantly improved overall survival with many animals showing complete tumor regression. Surviving animals with fully regressed tumors rejected newly implanted tumors when rechallenged with RENCA cells, indicating that combination treatment of SG-3$00802+$ anti-PD-1 generates long lasting anti-tumor memory responses.

Conclusions Microbiome interacts with the human immune system to impact anti-tumor immunity. Microbiome-derived peptides identified by Second Genome's discovery platform sg4sight modulate innate immune cells e.g. dendritic cells to promote anti-tumor immunity. SG-3-00802 peptide has the greatest potential to impact subjects who are potentially going to be non-responders to anti-PD-1 or have derived inadequate response to ICI therapies.

http://dx.doi.org/10.1136/jitc-2021-SITC2021.569 